\title{
Design of an Efficient Miniaturized UHF Planar Antenna
}

\author{
Kamal Sarabandi, Fellow, IEEE, and Reza Azadegan, Student Member, IEEE
}

\begin{abstract}
In this paper, the design aspects and the measured results of a novel miniaturized planar antenna are described. Such architectural antenna design is of great importance in mobile military communications where low visibility and high mobility are required. Slot radiating elements, having a planar geometry and capable of transmitting vertical polarization when placed nearly horizontal, are appropriate for the applications at hand. Slot antennas also have another useful property, so far as impedance matching is concerned. Basically, slot dipoles can easily be excited by a microstrip line and can be matched to arbitrary line impedances simply by moving the feed point along the slot. Antenna miniaturization can be achieved by using a high permittivity or permeability substrate and superstrate materials and/or using an appropriate antenna topology. Here, we demonstrate miniaturization by designing an appropriate geometry for a resonant narrow slot antenna. A very efficient radiating element that occupies an area as small as $0.12 \lambda_{0} \times 0.12 \lambda_{0}$ is designed and tested. Simulation results, as well as the measured input impedance and radiation patterns of this antenna, are presented. This structure shows a measured gain of $0.5 \mathrm{dBi}$ on FR4 substrate, which has a loss-tangent of the order of 0.01 . Also, the effect of finite ground plane size on gain and resonant frequency is investigated experimentally.
\end{abstract}

Index Terms-Miniaturized antennas, small antennas, slot antennas.

\section{INTRODUCTION}

$\mathbf{W}$ ITH THE advent of wireless technology and ever increasing demand for high data rate mobile communications the number of radios on military mobile platforms has reached a point that the available real estate for these antennas has become a serious issue. Similar problems are also emerging in the commercial sector where the number of wireless services planned for future automobiles, such as FM and CD radios, analog and digital cell phones, GPS, keyless entry, etc., is on the rise.

To circumvent the aforementioned difficulties to some extent, antenna miniaturization and/or compact multifunctional antennas must be considered. The subject of antenna miniaturization is not new. Literature concerning this subject dates back to the early 1940s [1], [2]. To our knowledge, the fundamental limitations of small antennas were first addressed by Chu in 1948 [2]. Using a multipole expansion and a clever equivalent

Manuscript received February 6, 2001; revised April 4, 2002. This work was supported in part by the US Army Research Office under contract DAA-99-1-0197 and by CACI Technologies, Inc.

The authors are with the Radiation Laboratory, Department of Electrical Engineering and Computer Science, The University of Michigan, Ann Arbor, MI 48109-2122 USA (saraband@eecs.umich.edu; azadegan@ eecs.umich.edu).

Digital Object Identifier 10.1109/TAP.2003.812239 circuit model, Chu was able to derive the $\mathrm{Q}$ factor of the equivalent circuit for each spherical mode in terms of the normalized radius $(\mathrm{a} / \lambda)$ of the smallest sphere enclosing the antenna. In [2] it is also shown that the Q of the lowest order mode is a lower bound for the $\mathrm{Q}$ of a single resonant antenna. This subject was revisited by Wheeler [3], Harrington [4], and Collins [5]. In [5], a similar procedure is used for characterization of a small dipole antenna using cylindrical wave functions. Then a cylindrical enclosing surface is used which produces a tighter lower bound for the Q of small antennas with large aspect ratios such as dipoles and helical antennas. Qualitatively, these studies show that for single resonant antennas, the smaller the maximum dimension of an antenna, the higher is its Q or, equivalently, the lower is its bandwidth [6]. However, in these papers no discussion is provided about miniaturization methods, antenna topology, or impedance matching.

Considering wave propagation where line-of-sight communication is an unlikely event, such as in an urban environment or over irregular terrain, carrier frequencies at the HF-UHF band are commonly used. At these frequencies, there is considerable penetration through vegetation and buildings, wave diffraction around obstacles, and wave propagation over curved surfaces. However, at these frequencies, the size of efficient antennas are relatively large, and therefore, a large number of such antennas may not fit in the available space without the risks of mutual coupling and co-site interference. Efficient antennas require dimensions of the order of half a wavelength for single frequency operation. To cover a wide frequency range, broadband antennas may be used. However, dimensions of these antennas are comparable to or larger than the wavelength at the lowest frequency. Besides, depending on the applications, the polarization and the direction of maximum directivity for different wireless systems operating at different frequencies may be different and hence a single broadband antenna may not be sufficient. It should also be noted that any type of broadband antenna is highly susceptible to electronic warfare jamming techniques. Variations of monopole and dipole antennas in use today are prohibitively large and bulky at HF through VHF.

In this paper, the topology of an efficient, miniaturized, resonant slot antenna is presented, and then its radiation, input impedance, and bandwidth characteristics are investigated. This class of antennas can simultaneously exhibit band selectivity and antijam characteristics in addition to possessing a planar structure and low profile, which is easily integrable with other RF and microwave circuits.

In what follows, we first investigate the design aspects of a miniaturized resonant slot spiral (coiled dipole) at low frequencies. It is shown that although a very good impedance match can be achieved, these antennas do not radiate efficiently. Then, 


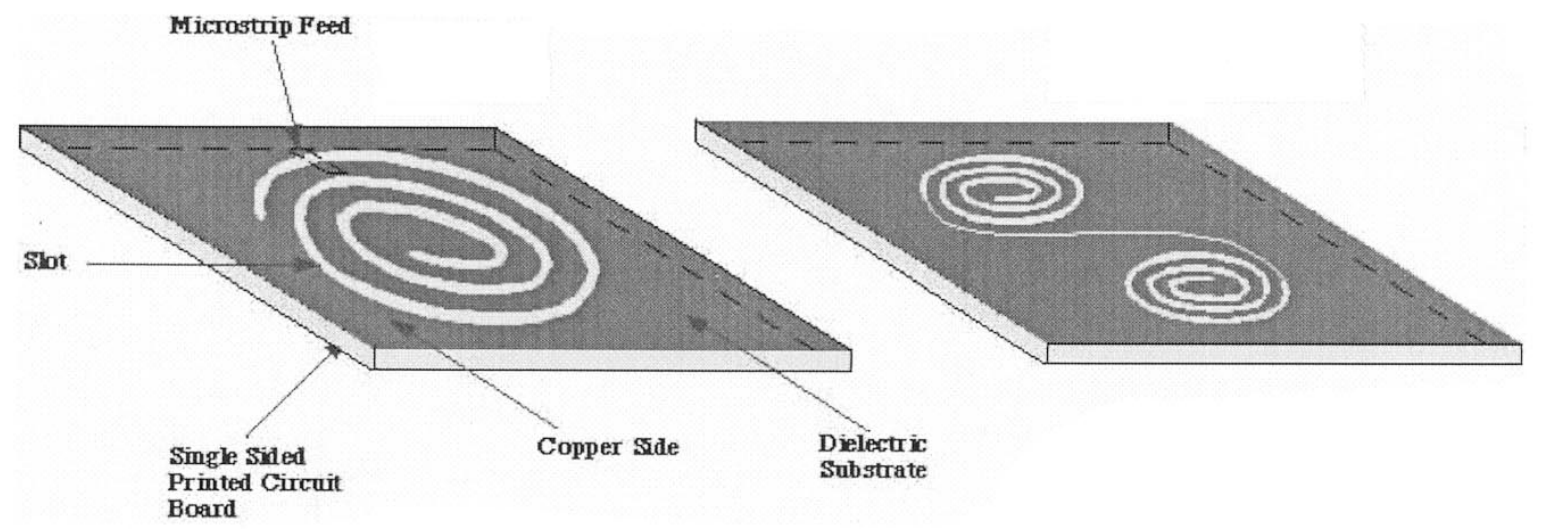

(a)

(b)

Fig. 1. The geometry of an (a) equiangular and (b) cornu resonant spiral antennas.

an alternate novel design for the construction of an efficient miniature antenna is considered, and the design aspects, in addition to the simulated results, are described. In Section III, the measured radiation characteristics of a prototype miniaturized antenna operating around $600 \mathrm{MHz}$ is presented to validate the design procedures and simulations. Finally the effect of the finite size ground plane on this type of slot antenna is addressed.

\section{Design OF A Miniaturized UHF ANTENNA}

Equiangular and Archimedean spiral antennas [7], [8] are commonly used as broadband antennas. These antennas usually have an arm length larger than a wavelength. Not much has been reported about the performance of spiral antennas when the arm length is half of the guided wavelength (a resonance condition). One obvious reason is that slot spiral antennas are usually center fed where at the first resonance the input impedance is very high and impedance matching becomes practically impossible. However, if we allow the feed point to be moved to one end of the arm, impedance matching to transmission lines with typical characteristic impedances seems possible.

First, an equiangular spiral slot, as shown in Fig.1 (a), is considered. Using electrostatic analysis, the guide wavelength of a slot line with finite ground plane was calculated. The calculated guide wavelength was used to design an arm length of $\lambda_{\mathrm{g}} / 2$. The spiral was fed from the outer end using a microstrip line. The location of feed point along the arm was found by trial and error until a good match was achieved. Also, a slot dipole $(\lambda / 2)$ coil around two center points (cornu spiral) as shown in Fig.1 (b) was considered. For this antenna, the microstrip feed was coiled around one arm on the other side of the substrate (over the ground plane) in order to feed the slot near an end point. Again by trial and error the location of feed point for best impedance match was found. A return loss of better than $20 \mathrm{~dB}$ for these antennas was obtained. However, gain measurement of these antennas showed poor radiation efficiencies (less than $-10 \mathrm{~dB}$.) The efficiency of the cornu spiral was higher than the simple spiral antenna, however, neither showed an acceptable level of antenna efficiency. Far-field pattern and gain measurements at $60 \mathrm{MHz}$ were carried out in an outdoor slant range using a boom truck and two antenna positioners.
The main problem in these designs seems to stem from the fact that the far-field generated by opposing equivalent magnetic currents along a resonant spiral antenna tend to cancel. To investigate whether ohmic losses are responsible for the low antenna efficiency, a similar antenna having the same geometry and substrate dielectric constant were constructed using a different dielectric quality factor and copper thickness. It was found that ohmic losses were not responsible for the lack of antenna efficiency to the degree observed in our measurements. By putting absorbers around the feed cable and antenna positioner, it was found that these antennas produce a very strong near field which can excite current on the nearby objects, such as the cable feeding them and the antenna positioner. The current induced on these objects in turn radiated the input power, but not necessarily in the direction of the maximum antenna radiation pattern.

A different topology for a miniaturized resonant slot dipole is sought that does not have the drawbacks of the previously discussed spiral and cornu geometries. A major reduction in size is achieved by noting that a slot dipole can be considered as transmission line resonator where at the lowest resonant frequency the magnetic current (transverse electric field in the slot) goes to zero at each end of the dipole antenna. As mentioned before at this frequency the antenna length $l=\lambda_{\mathrm{g}} / 2$ where $\lambda_{\mathrm{g}}$ is the wavelength of the quasi-TEM mode supported by the slot line. $\lambda_{\mathrm{g}}$ is a function of substrate thickness, dielectric constant, and the slot width, which is shorter than the free-space wavelength. In view of transmission line resonators, one can also make a quarter-wave resonator by creating a short circuit at one end and an open circuit at the other end. However, creating a physical open circuit for slot lines is not practical. The new design borrows the idea of nonradiating tightly coiled slot spiral from the previous design. Basically, a spiral slot of a quarter wavelength and shorted at one end behaves as an open circuit at the resonant frequency. Therefore, a quarter-wave slot line short-circuited at one end and terminated by the nonradiating quarter-wave spiral should resonate and radiate electromagnetic waves very efficiently. With this topology the size of the slot dipole can be reduced by approximately $50 \%$. Further reduction can be accomplished by bending the radiating section. This bending procedure should be done so that no section of the resulting line geometry carries a magnetic current 


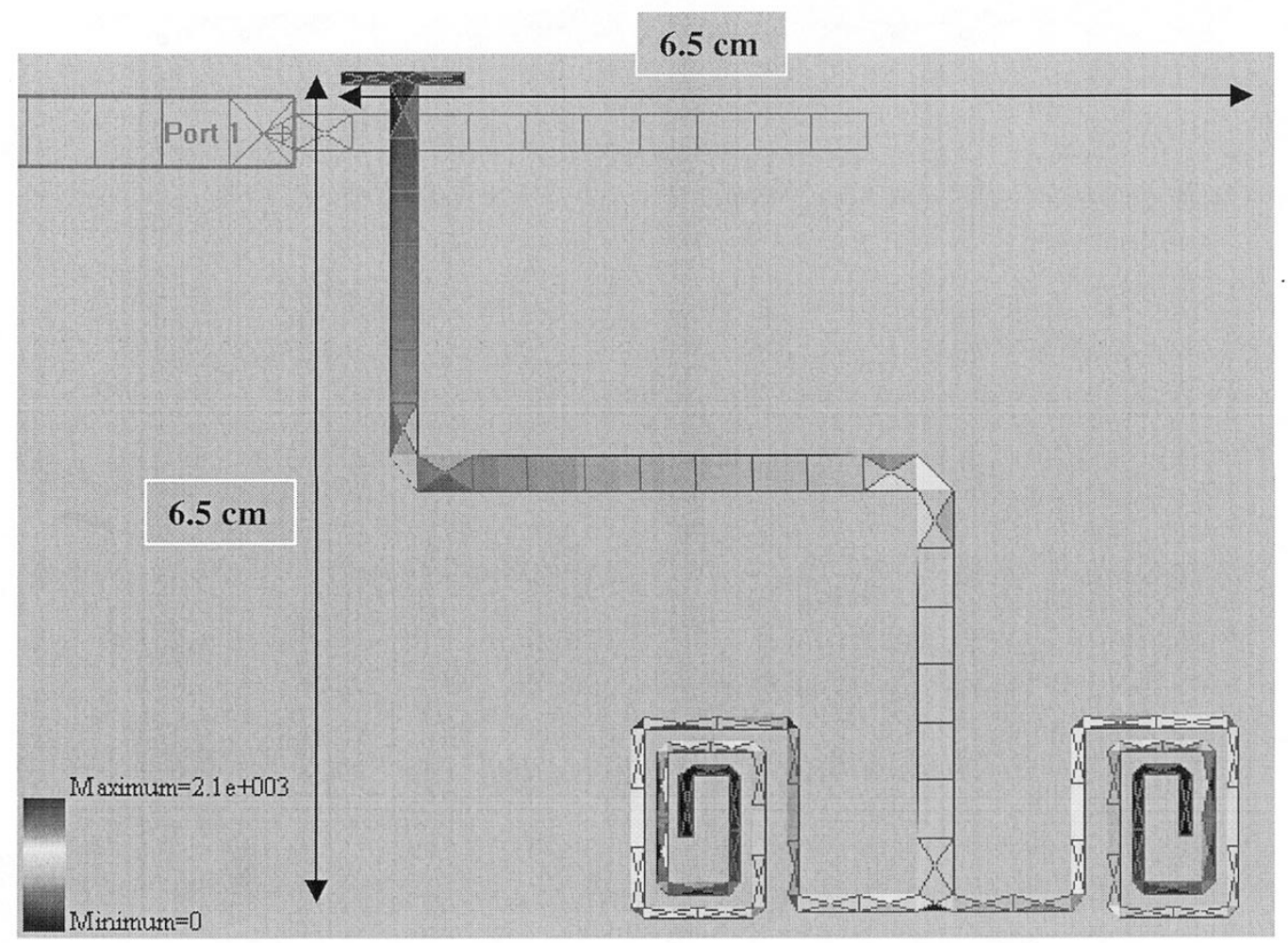

Fig. 2. Magnetic current distribution on the UHF miniaturized slot antenna at the resonance frequency $(600 \mathrm{MHz})$. The figure shows the ground-plane side of the antenna and the meshing configuration used in the method of moments calculations. PiCASSO software was used in these calculations.

opposing the current on any other sections. Fig. 2 shows the geometry of a typical $\lambda_{\mathrm{g}} / 4$ compact resonating slot antenna. The radiating section is terminated with two identical quarter-wave nonradiating spiral slots to maintain the symmetry. It was found that by splitting the magnetic current at the end into equal and opposing magnetic currents the radiation efficiency is enhanced. Since the magnetic current distribution attains its maximum at the end of the quarter-wave line, the magnetic current in the beginning segments of a single (unbalanced) quarter-wave spiral reduces the radiation of the radiating section. But the opposite magnetic currents on two such spirals simply cancel the radiated field of each other, and as a result, the radiated field of the radiating section remains intact. Some additional size reduction can also be achieved, by noting that the strength of the magnetic current near the short-circuited end of the radiating section is insignificant. Hence, bending this section of the line does not significantly reduce the radiation efficiency despite allowing opposing currents. In Fig. 2 the T-top represents a small reduction in length of the line without affecting the radiation efficiency. This antenna is fed by an open ended microstrip line. A quarter wavelength line corresponds to a short-circuit line under the slot. However, using the length of the microstrip line as an adjustable parameter, the reactive part of the antenna input impedance can be compensated for.

Figs. 2 and 3, respectively, show the electric current distribution on the microstrip feed and the magnetic current distribution on the slot of the compact UHF antenna designed to operate at $600 \mathrm{MHz}$. For this design, we chose an ordinary FR4 substrate with thickness of $3 \mathrm{~mm}$ (120 mil) and dielectric constant $\varepsilon_{\mathrm{r}}=4$. PiCASSO software was used for the simulations of this antenna
[9]. The microstrip feed is constructed from two sections: 1) a $50 \Omega$ line section and 2) an open-ended $80 \Omega$ line. The $80 \Omega$ line is thinner which allows for compact and localized feeding of the slot. The length of this line is adjusted to compensate for the reactive component of the slot input impedance. Noting that the slot appears as a series load in the microstrip transmission line, a line length of less than $\lambda_{\mathrm{m}} / 4$ compensates for an inductive reactance and a line length of longer than $\lambda_{\mathrm{m}} / 4$ compensates for a capacitive reactance. Here, $\lambda_{\mathrm{m}}$ is the guided wavelength on the microstrip line. First, a quarter wavelength section was chosen for the length of the microstrip line feeding the slot. In this case, the simulation predicts the impedance of the slot antenna alone. Through this simulation, it was found that the slot antenna fed near the edge is inductive. So, a length less than $\lambda_{\mathrm{m}} / 4$ is chosen for the open-ended microstrip line to compensate for the inductive load. The real part of input impedance of a slot dipole depends on the feed location along the slot and increases from zero at the short-circuited end to about $2000 \Omega$ at the center (quarter wavelength from the short circuit). This property of the slot dipole allows for matching to almost all practical transmission lines. The crossing of the microstrip line over the slot was determined using the full-wave analysis tool, (PiCASSO) and by trial-and-error. The uniform current distribution over the $50 \Omega$ line section indicates no standing wave pattern, which is a result of a very good input impedance match.

Apart from the T-top section, the quarter-wave radiating section of the slot dipole is composed of three slot line sections, two vertical and one horizontal. Significant radiation emanates from the middle and lower sections. Polarization of the antenna can be chosen by changing the relative size of these two sections. 


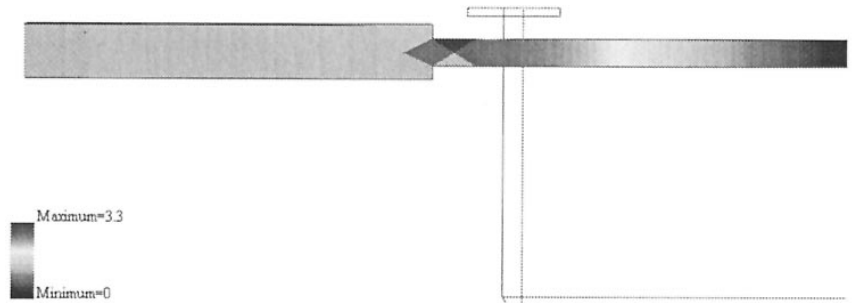

Fig. 3. Electric current distribution on the microstrip feed of the slot antenna at the resonant frequency.

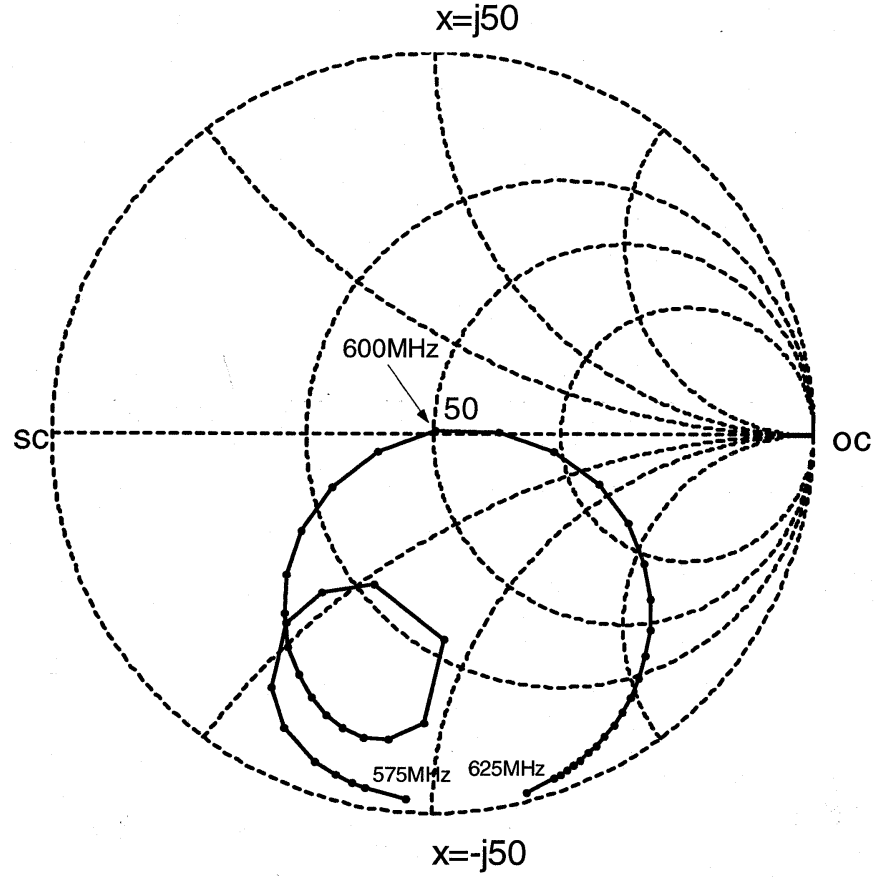

(a)

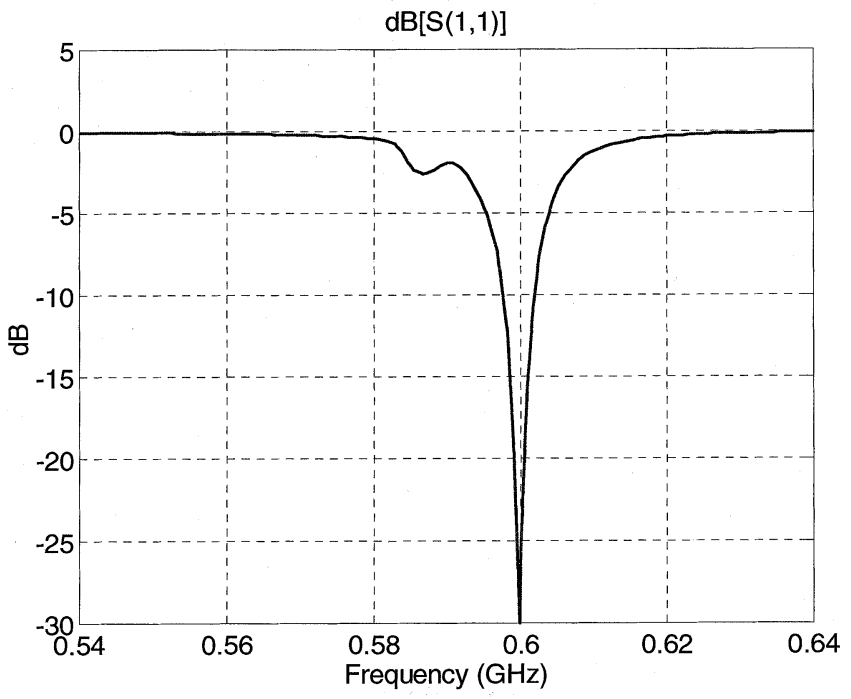

(b)

Fig. 4. Simulated reflection coefficient of the miniaturized UHF antenna on an infinite ground plane. (a) Smith chart representation. (b) Magnitude of $\left|\mathrm{S}_{11}\right|$ in logarithmic scale.

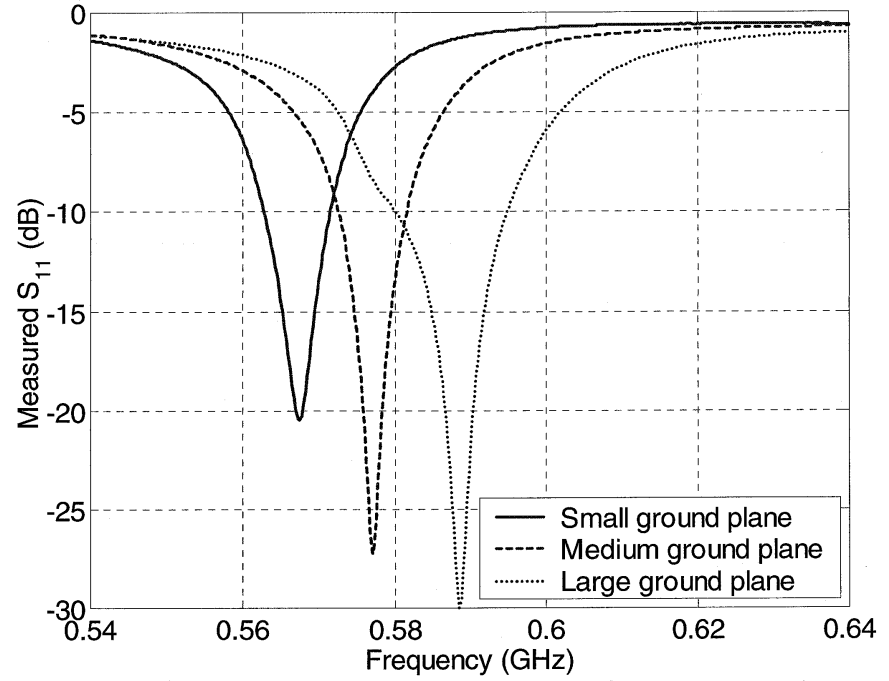

Fig. 5. Measured magnitude of reflection coefficient of three miniaturized UHF slot antennas, described in Table I and shown Fig. 7, all having the same size and geometry, but with a different ground plane sizes.

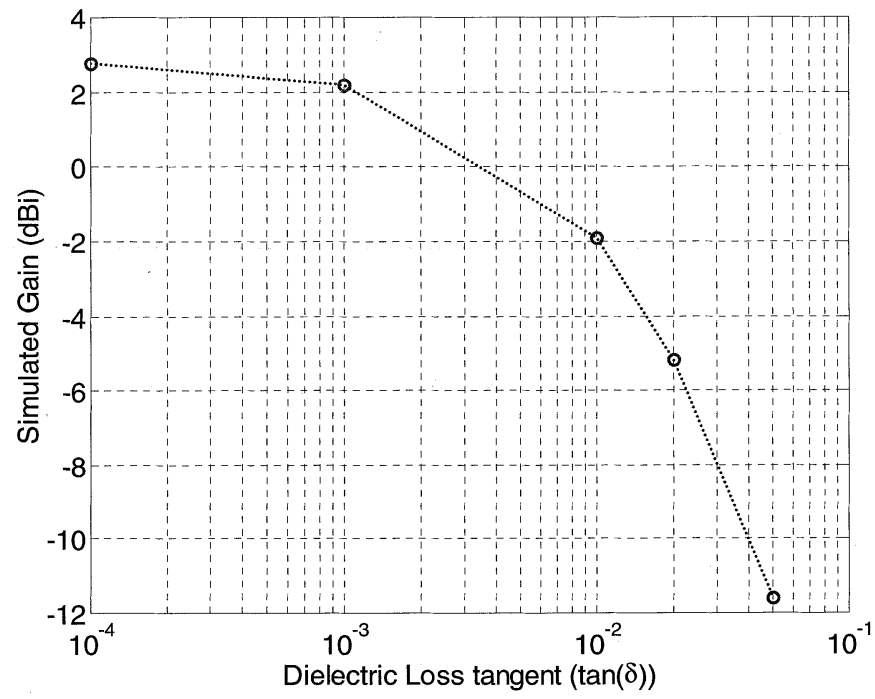

Fig. 6. Simulated gain of the UHF miniaturized antenna on an infinite substrate with $\epsilon_{\mathrm{r}}=4.0(1-j \tan \delta)$.

In this design the relative lengths of the three line sections were chosen in order to minimize the area occupied by the slot structure. The slot width of the first section can be varied in order to obtain an impedance match as well. When there is a limitation in moving the microstrip and slot line crossing point, the slot width may be changed. At a given point from the short-circuited end, an impedance match to a lower line impedance can be achieved when the slot width is narrowed. This was used in this design, as the slot line width of the top vertical section is narrower than the other two sections. It should be pointed out that, by narrowing the slot line width, the magnetic current density increases but the total magnetic current in the line does not. In other words, there is no discontinuity in the magnetic current along the line at points where the slot width is changed, however, there are other consequences. One is the change in the char- 


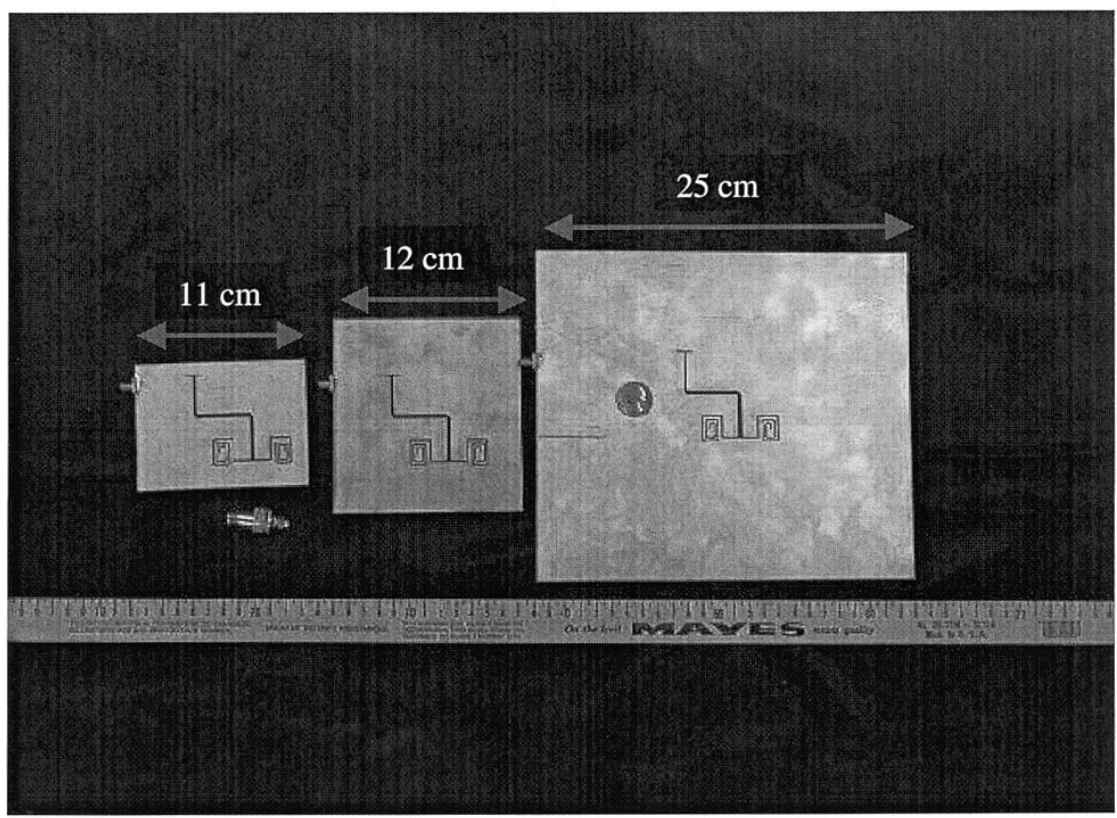

Fig. 7. A photograph of three miniaturized UHF antennas. As shown, all three slot antennas have the same geometry and dimensions. The only difference is the size of the ground.

acteristic impedance of the line, and the second is the change in the antenna efficiency, considering the finite conductivity of the ground plane. There are two components of electric current flowing on the ground plane, one component flows parallel to the edge and the other is perpendicular. For narrow slots, the current density of the parallel component near the edge goes up, and as a result, this current sees a higher ohmic resistance. The magnetic current over the T-top section is very low and does not contribute to the radiated field but its length affects the resonant frequency. Half the length of the T-top section originally was part of the first vertical section, which is removed and placed horizontally to lower the vertical extent of the antenna.

The slot line sections were chosen so that a resonant frequency of $600 \mathrm{MHz}$ was achieved. At this frequency the slot antenna occupies an area of $(6.5 \mathrm{~cm} \times 6.5 \mathrm{~cm})$ or in terms of the free-space wavelength $0.12 \lambda_{0} \times 0.12 \lambda_{0}$. Fig. 4 (a) and (b), respectively, show the simulated input impedance and return loss of the miniaturized UHF antenna as a function of frequency. It is shown that the $1.2 \mathrm{VSWR}(-10 \mathrm{~dB}$ return loss) bandwidth of this antenna is around $6 \mathrm{MHz}$, which corresponds to a $1 \%$ fractional bandwidth. This low bandwidth is characteristic of miniaturized and resonant slot dipoles. The simulation also shows a weak resonance, which may be caused by the interaction between the radiating element and the nonradiating spirals. In fact, careful examination of the magnetic current distributions over the nonradiating spirals shows the asymmetry caused by the near field interaction of the radiating element with the nonradiating spirals.

The polarization of this antenna may appear to be rather unpredictable at a first glance due to its convoluted geometry. However, it can be conjectured that the polarization of any miniaturized antenna whose dimensions are much smaller than a wavelength cannot be anything other than linear. This is basically because of the fact that the small electrical size of the antenna does not allow for a phase shift between two orthogonal
TABLE I

RESONANT FREQUENCIES GaINS AND THE GROUND Plane Sizes of THREe IDENTICAL UHF MINIATURIZED SLOT ANTENNAS. HERE THE EFFECT OF Ground Plane Size on the Resonant FreQuency and ANTENNA GAIN IS DEMONSTRATED

\begin{tabular}{c|c|c|c}
\hline & Ground Plane Size & Resonant Frequency & Gain $(\mathrm{dBi})$ \\
\hline Antenna 1 & $8.5 \mathrm{~cm} \times 11 \mathrm{~cm}$ & $568 \mathrm{MHz}$ & -5.0 \\
\hline Antenna 2 & $12 \mathrm{~cm} \times 13 \mathrm{~cm}$ & $577 \mathrm{MHz}$ & -2.0 \\
\hline Antenna 3 & $22.5 \mathrm{~cm} \times 25 \mathrm{~cm}$ & $592 \mathrm{MHz}$ & 0.5 \\
\hline
\end{tabular}

components of the radiated field required for producing an elliptical polarization. Hence, by rotating the antenna a desired linear polarization along a given direction can be obtained.

\section{REALIZATION AND MEASUREMENTS}

An antenna based on the layout shown in Figs. 2 and 3 was made on a FR4 printed-circuit-board. In the first realization, the size of the ground plane was chosen to be $8.5 \mathrm{~cm} \times 11 \mathrm{~cm}$. The return loss of this antenna was measured with a network analyzer and the result is shown by the solid line in Fig. 5. It is noticed that the resonant frequency of this antenna is at $568 \mathrm{MHz}$, which is significantly lower than what was predicted by the simulation. Also, the measured return loss for the designed microstrip feed line (not shown here) was around $-10 \mathrm{~dB}$. To get a better return loss the length of the microstrip line had to be extended slightly. Fig. 5 shows the measured return loss after the modification. The gain of this antenna was also measured against a calibrated antenna. Under a polarization matched condition a gain of $-5.0 \mathrm{dBi}$ (gain in $\mathrm{dB}$ against an isotropic radiator) is measured. The simulated gain value of this antenna using an infinite ground plane and $\epsilon=4.0-j 0.0$ is found to be $2.8 \mathrm{dBi}$. The difference in the simulation results and the measured ones can be attributed to the finiteness of the ground plane, finite conductivity of the ground plane, and 


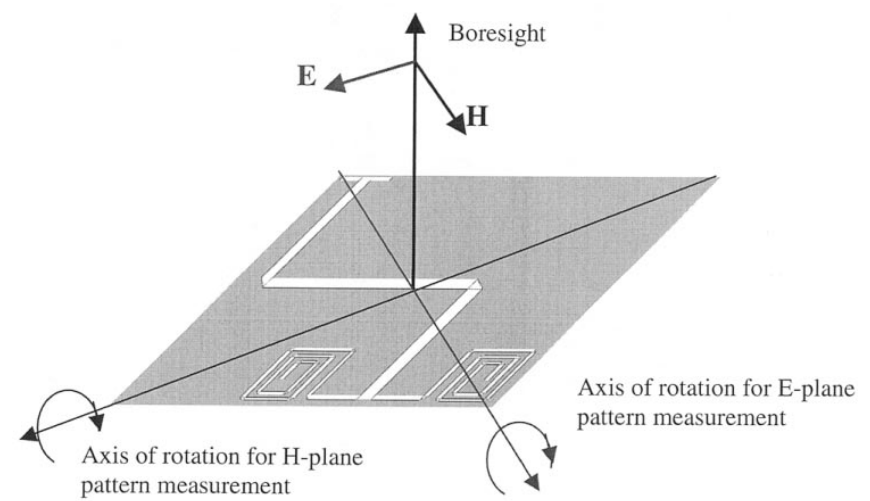

Fig. 8. E- and H-planes of the antenna under test characterized experimentally. Co- and cross-polarized pattern measurements are performed in these two principal planes.

the loss tangent of the substrate. The effect of the imaginary part of the substrate dielectric constant $\left(\epsilon=4.0-j \epsilon^{\prime \prime}\right)$ can be quantified using a numerical simulation. Fig. 6 shows the simulated gain values of this antenna as a function of $\epsilon^{\prime \prime}$ with an infinite ground plane. It is shown that, as expected, the gain is decreased when the loss tangent is increased. Hence, it is very important to use substrates with a very low loss tangent. The FR4 used for this antenna has a loss tangent $(\tan \delta \approx 0.01)$ at UHF. To investigate the effect of ground plane size on the resonance frequency and radiation efficiency, two more antennas having the same geometry and dimensions but with different ground plane sizes were made. The measured resonant frequencies are also shown in Fig. 5. Fig. 7 shows the photograph of these antennas. The dimensions of the ground planes and the measured gain of these antennas are reported in Table I. As expected the resonant frequency and the gain of the antenna approaches predicted values as the size of the ground plane is increased. The gain of Antenna 3 is almost as high as the gain of an ideal dipole considering the loss tangent of the substrate used in these experiments.

The gain reduction as a function of ground plane size can be explained by noting that the equivalent magnetic currents that flow in the upper and lower side of the ground plane are in opposite directions. In the case of infinite ground plane, the upper and lower half spaces are electromagnetically decoupled. However, when the ground plane is finite and small compared to the wavelength the radiated field from the lower half space can reduce the radiated field from the magnetic current in the upper half space. The level of back radiation depends on the size of the ground plane; that is, the smaller the ground plane the higher the back radiation. Ignoring the substrate $\left(\varepsilon_{\mathrm{r}}=1\right)$, radiation from the upper and lower magnetic currents completely cancel each other in the plane of the perfect conductor (creates a null in the radiation pattern). However, because of the substrate and depending on its thickness and relative dielectric constant, a perfect cancellation does not occur. This explains the discrepancies observed between the measured and predicted radiation patterns (for infinite ground plane). Also, there are strong edge currents on the periphery of a finite ground which decrease as the size of the ground plane is increased. The confined currents around

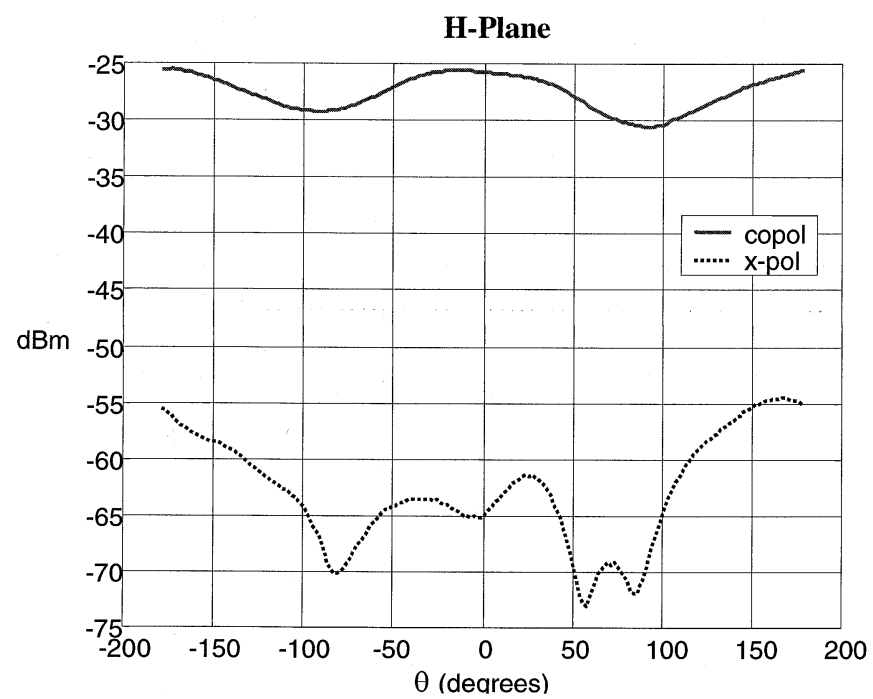

(a)

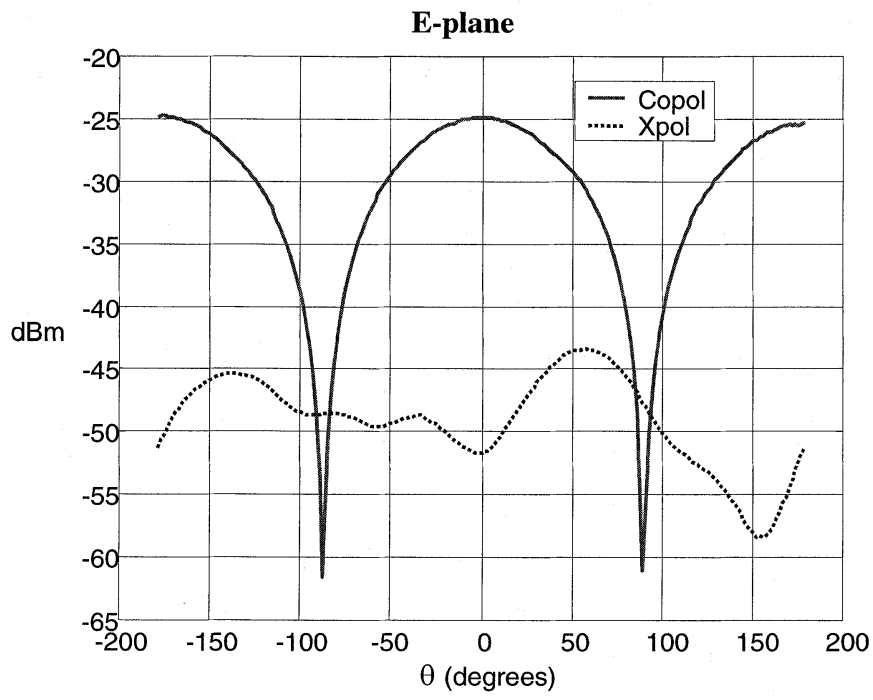

(b)

Fig. 9. Co- and cross-polarized pattern of the miniaturized UHF antenna in (a) H-plane and (b) E-plane.

the edge experience an ohmic loss which is responsible for the decrease in the antenna gain.

The radiation patterns of these antennas were also measured at the University of Michigan anechoic chamber. A linearly polarized antenna was used as the reference. First, the polarization of the antenna was determined at the direction of maximum radiation (normal to the ground plane). Then by rotating the antenna under test about the direction of maximum radiation, it was found that indeed the polarization of the miniaturized antenna is linear. Fig. 8 shows the direction of maximum radiation, the direction of electric filed (polarization), and magnetic field at the antenna boresight. Figs.9 (a) and (b) show the co- and cross-polarized antenna patterns in the $\mathrm{H}$ - and E-planes, respectively. It is shown that the antenna polarization remains linear on these principal planes. As discussed before, the E-plane gain in the plane of the ground plane $\left(\theta=90^{\circ}\right)$ drops because of the finiteness of the ground plane. If the substrate were to be removed, the 
E-plane gain in the plane of the conductor would drop to zero. Hence, having a thick substrate helps achieve a more uniform pattern. Thick and high permittivity substrate also increases front-to-back radiation ratio. Since our substrate thickness is only a small fraction of the wavelength, almost similar gain values are measured in the upper and lower half spaces.

It is worth mentioning that further miniaturization can easily be accomplished by increasing the dielectric constant of the substrate. In this case, the guide wavelength shortens which in turn allows for a smaller antenna. As mentioned in Section I, further antenna miniaturization is accompanied by a reduction of the antenna bandwidth. Also, confining the electric currents on the ground plane into a smaller area results in a higher ohmic loss or equivalently lower antenna efficiency.

\section{CONCLUSION}

A novel topology for designing an electrically small resonant slot antenna is demonstrated. A major size reduction was achieved by constructing a $\lambda_{\mathrm{g}} / 4$ resonant slot rather than the traditional $\lambda_{\mathrm{g}} / 2$ antenna. This is accomplished by generating a virtual open circuit at one end of the slot. Further miniaturization was achieved by bending the slot into three pieces in order to use the area of the board more efficiently. The antenna geometry occupies a very small area $\left(0.014 \lambda_{0}{ }^{2}\right)$ of a PC board with $\varepsilon_{\mathrm{r}}=4.0$ and thickness $3 \mathrm{~mm}$. The antenna is very efficient and shows a gain as high as a dipole antenna and a $1 \%$ bandwidth. It is also shown that if the antenna is made on a small ground plane its gain will be reduced and its radiation pattern changes slightly.

\section{REFERENCES}

[1] H. A. Wheeler, "Fundamental limitations of small antennas," Proc. IRE, vol. 35, pp. 1479-1484, Dec. 1947.

[2] L. J. Chu, "Physical limitations of omni-directional antennas," J. Appl. Phys., vol. 19, pp. 1163-1175, Dec. 1948.

[3] H. A. Wheeler, "Small antennas," IEEE Trans. Antennas Propagat., vol. AP-23, pp. 462-469, July 1975.

[4] R. F. Harrington, "Effect of antenna size on gain, bandwidth, and efficiency," J. Res. Nat. Bur. Stand., vol. 64D, pp. 1-12, 1960.

[5] R. E. Collin, "Small antennas," IEEE Trans. Antennas Propagat., vol. AP-12, pp. 23-27, Jan. 1964

[6] R. C. Hansen, "Fundamental limitations in antennas," Proc. IEEE, vol. 69, pp. 170-182, Feb. 1981.

[7] J. A. Kaiser, "The Archimedean two-wire spiral antenna," IRE Trans. Antennas Propagat., vol. AP-8, pp. 312-323, May 1960.

[8] P. E. Mayes, "Planar and other wide-angle logarithmic spiral over ground," Electromagn., vol. 14, pp. 329-362, 1994.

[9] em PiCASSO Pro., EMAG Technologies, Inc., Ann Arbor, MI.

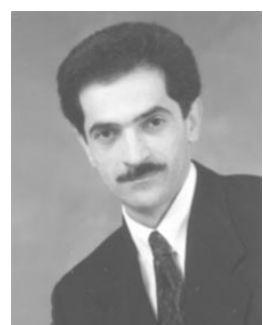

Kamal Sarabandi (S'87-M'90-SM'92-F'00) received the B.S. degree in electrical engineering from Sharif University of Technology, Tehran, Iran, and the M.S. degrees in electrical engineering and mathematics and the Ph.D. degree in electrical engineering, all from The University of Michigan, Ann Arbor, in 1980, 1986, and 1989, respectively

$\mathrm{He}$ is the Director of the Radiation Laboratory and a Professor in the Department of Electrical Engineering and Computer Science at the University of Michigan. His research areas of interest include microwave and millimeter-wave radar remote sensing, electromagnetic wave propagation, and antenna miniaturization. He has 20 years of experience with wave propagation in random media, communication channel modeling, microwave sensors, and radar systems, and is leading a large research group including two research scientists, ten Ph.D. degree students, and two M.S. degree students. Over the past ten years, he has graduated $14 \mathrm{Ph} . \mathrm{D}$. degree students. He has served as the Principal Investigator on many projects sponsored by the National Aeronautics and Space Administration, ASA, JPL, the Army Research Office, the Office of Naval Research, ARL, the National Science Foundation, DARPA, and numerous industries. He has published many book chapters and more than 95 papers in refereed journals on electromagnetic scattering, random media modeling, wave propagation, antennas, microwave measurement techniques, radar calibration, inverse scattering problems, and microwave sensors. He has also had more than 200 papers and invited presentations in many national and international conferences and symposia on similar subjects.

Dr. Sarabandi is a Vice President of the IEEE Geoscience and Remote Sensing Society (GRSS), Chairman of the Awards Committee of the IEEE GRSS, and a Member of IEEE Technical Activities Board Awards Committee. He is currently serving as the Associate Editor of the IEEE TRANSACTIONS ON ANTENNAS AND PROPAGATION and the IEEE SENSORS JouRnAL. He is also a Member of Commission F of URSI and of The Electromagnetic Academy. He is listed in American Men \& Women of Science Who's Who in America and Who's Who in Electromagnetics. He was the recipient of the prestigious Henry Russel Award from the Regent of The University of Michigan (the highest honor the University of Michigan bestows on a faculty member at the assistant or associate level). In 1999, he received a GAAC Distinguished Lecturer Award from the German Federal Ministry for Education, Science, and Technology given to about ten individuals worldwide in all areas of engineering, science, medicine, and law. $\mathrm{He}$ was also a recipient of a 1996 Teaching Excellence Award from the EECS Department of The University of Michigan. In the past several years, joint papers presented by his students at a number of symposia (IEEE AP'95,'97,'00,'01, IEEE IGARSS'99, IEEE MTTS'01) have received student paper prize awards.

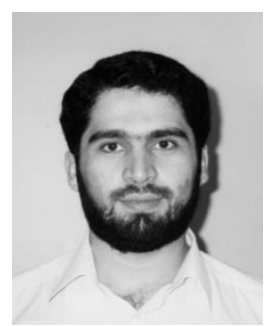

Reza Azadegan (S'98) was born in Tehran, Iran, in 1974. He received the B.S degree in electrical engineering from Sarif University of Technology, Tehran, Iran, and the M.S. degree in electrical engineering from K. N. Toosi University of Technology, Tehran, Iran, in 1996 and 1998, respectively. $\mathrm{He}$ is currently working toward the Ph.D. degree in the Radiation Laboratory at the University of Michigan, Ann Arbor, where he is working on the miniaturization of planar antennas and microwave filters for wireless communication systems.

From 1997 to 1999, he was with the Computational Electromagnetic Laboratory, Sharif University of Technology, as a Graduate Researcher working on the optimal design of parabolic reflector antennas using high frequency techniques and genetic algorithms and electromagnetic wave propagation in optical waveguides. 